\title{
Adriamycine-Induced Cardiomyopathy
}

\author{
Marios Lemoniatis
}

\begin{abstract}
Adriamycin is an antineoplastic agent used in a wide variety of malignancies. Its use is limited because of a cumulative, dose-dependent irreversible cardiomyopathy. We report a case of adriamycin-induced cardiomyopathy, after he had undergone chemotherapy for Hodgin lymphoma. The patient had no cardiac risk factors, and neither laboratory nor imaging findings suggested myocarditis or dilated cardiomyopathy. Echocardiographic findings and differential diagnosis led us to attribute his condition to subacute doxorubicin-induced cardiomyopathy.
\end{abstract}

Keywords: Anthracyclines; Cardiomyopathy; Adriamycin; Hodgin

\section{Introduction}

Adriamycin belongs to the group of anthracyclines which were introduced for the use of cancer treatment in the late 1960s [1]. Anthracyclines are derived from various types of streptomycin. They are red in color, which derives the synthetic "rubi" used in medicines (doxorubicin) [2]. Their action produces oxygen and hydroxyl radicals [3], which are mainly responsible for cardiotoxicity in turn leading to cardiomyopathy. The prognosis for adriamycin-induced cardiomyopathy remains unfavorable despite the applied treatment $[4,5]$.

\section{Case Report}

A 54-year-old man was referred to the Cardiology Department of the Military Hospital due to shortness of breath, orthopnoea and fatigue which were gradually worsening throughout the month.

His medical history only mentioned that he had undergone

Manuscript accepted for publication April 10, 2015

Cardiology Department, National Guard Military Hospital, Cyprus.

Email: dr_mlemon@yahoo.com

doi: http://dx.doi.org/10.14740/jmc2132w chemotherapy for Hodgkin lymphoma in 2009.

The clinical examination revealed tachycardia, with a frequency of $115 \mathrm{bpm}$ and blood pressure of 110/70 $\mathrm{mm} \mathrm{Hg}$. Further there were rales on lung bases and mild systolic murmur. In the chest X-rays there were signs of increased dimension of the left ventricular and intensification of the lung vessels. The electrocardiogram revealed left bundle branch block (Fig. 1).

Echocardiography was performed which revealed dilatation of the left atrium and left ventricle $(6.6 \mathrm{~cm})$ with moderate mitral regurgitation, mild tricuspid insufficiency and left ventricular ejection fraction of $35 \%$. A 24-h Holter monitor was placed which revealed a few ventricular premature beats (Fig. 2,3).

Due to the low blood pressure that the patient had, a symptomatic treatment with low dose of diuretics, b-blocker and ACE inhibitor was started. A coronary angiography was performed which showed no coronary artery disease. Examining the medical records of Nicosia General Hospital, where the chemotherapy took place, an echocardiography study prior to the initiation of the chemotherapy was reported with normal ejection fraction. From all the above mentioned the conclusion was reached that the patient suffers from adriamycin-induced cardiomyopathy.

\section{Discussion}

The cardiotoxicity from adriamycin can appear as acute reversible myocarditis, subacute cardiomyopathy and chronic cardiomyopathy.

Acute myocarditis constitutes $11 \%$ [6] of all incidents and it can occur even with the issuance of only one dosage. It is expressed with chest pain, pulse feeling and ventricular or supraventricular arrhythmias. The treatment is supporting.

The subacute cardiomyopathy can occur 30 days from the last dose of the treatment and up to 10 years afterwards. The impact of cardiotoxicity is directly related to the dose of medicine, to the combination with other anticancer medicines, to the age of the patient and to the background history of cardiovascular diseases as hypertension and cardiac insufficiency of the patient.

Chronic cardiomyopathy occurs after many years (on average 14 years) [7] from the first day of the treatment and it is characterized by symptoms and signs of heart failure and ar- 


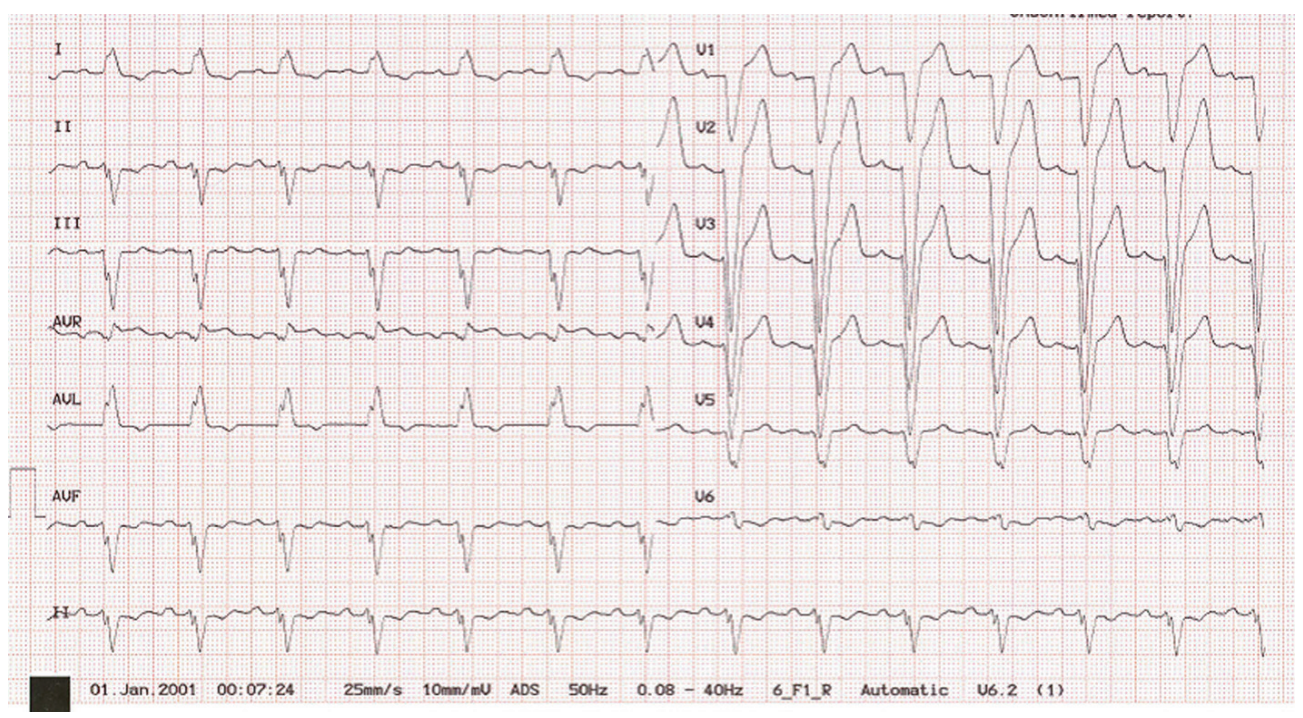

Figure 1. Electrocardiography of the patient after chemotherapy

rhythmias that did not exist at the end of treatment.

Various methods are applied for the early diagnosis of anthracycline-induced cardiotoxicity. Fundamental role has the receipt of a good medical history and the complete extensive clinical examination prior to the issuance of treatment. The servant doctor must be alert for a possible appearance of signs and symptoms of cardiac insufficiency. Even though the best method for diagnosing cardiotoxicity is the myocardial biopsy, the most reliable, non-invasive method for the diagnosis and follow-up of above patients is the estimate of ejection fraction of the left ventricle via echocardiography [8]. When after the issuing of the treatment the ejection fraction is under $45 \%$, it is considered that the patient has an increased risk of cardiotoxicity. On the contrary when it remains above $60 \%$ the issuing of treatment is considered sure. For ejection fraction between $45 \%$ and $60 \%$, smaller possible dose should be used, with frequent follow-up of the patient. In cases of doubt for the continuation of treatment, a myocardial biopsy was performed for determining the extent of myocardial damage

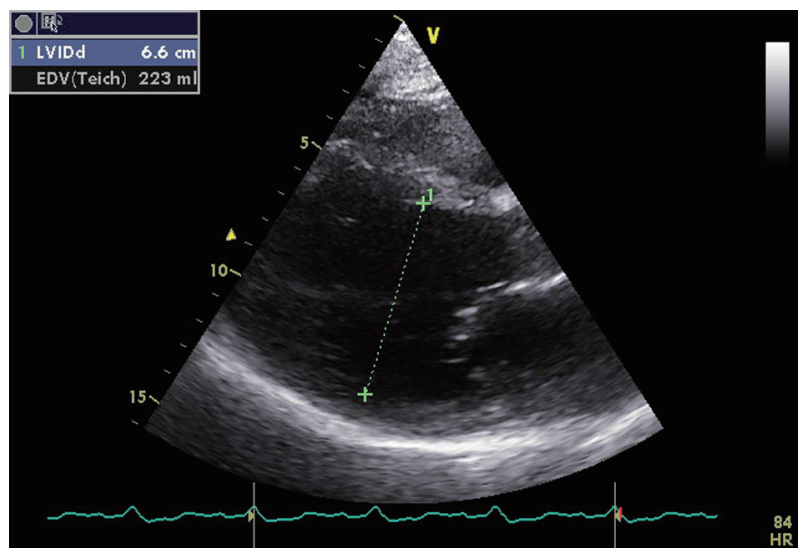

Figure 2. Echocardiography image that shows the dilatation of left ventricle after the chemotherapy.
[9].

All studies lead to the conclusion that the most important factor in the prevention of cardiomyopathy is the reduction of a whole engaged dose of the patient to $<450 \mathrm{mg} / \mathrm{m}^{2}$ [6]. It was proven that an important role in the prevention was the use of anthracyclines proportionals, the alternative methods of issuing of medicines and the slow continuous infusion.

There is no special treatment for anthracyclines-induced cardiomyopathy. The same medicines are used as for all other types of cardiomypathies. In patients with malignant arrhythmias amiodarone and implanting defibrillator should be used. The possibility of heart transplantation is a possibility for patients that have been cured completely from their disease [10].

Anthracycline-induced cardiomyopathy remains a lethal disease despite the intensive research that has been done in the sector of prevention and treatment. Due to the fact that a large number of patients begin to receive treatment from childhood, patients must be monitored throughout their whole life since cardiotoxicity can present itself even after many years.

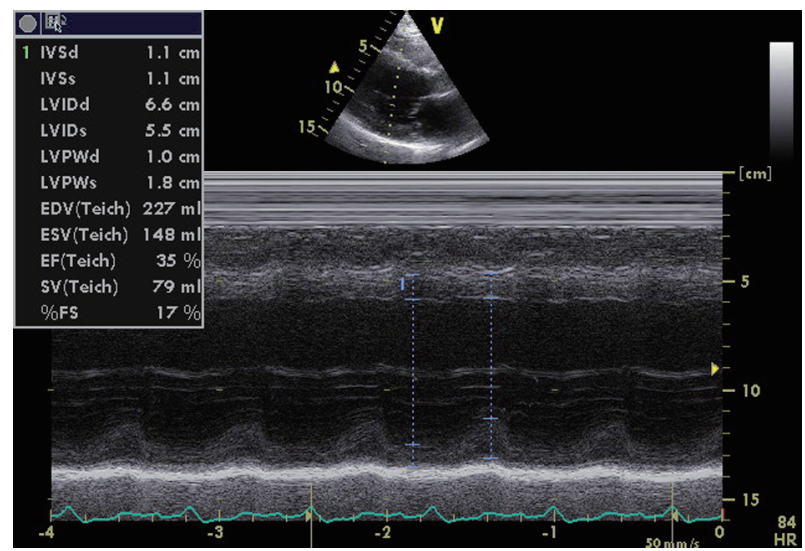

Figure 3. Echocardiograhy image that shows the poor ejection fraction of left ventricle after the chemotherapy. 


\section{References}

1. Fountzilas G, Barbounis B. Basic principles of cancer treatment. University Studio Press, Thessaloniki, 1997.

2. Dimarco A, Gaetani M, Orezzi P, Scarpinato BM, Silvestrini R, Soldati M, Dasdia T, et al. 'Daunomycin', a New Antibiotic of the Rhodomycin Group. Nature. 1964;201:706-707.

3. Sinha BK, Katki AG, Batist G, Cowan KH, Myers CE. Adriamycin-stimulated hydroxyl radical formation in human breast tumor cells. Biochem Pharmacol. 1987;36(6):793-796.

4. Jordon MA. Anti-cancer agents. Cur Med Chem. 2002;2:1-17.

5. Takemura G, Fujiwara H. Doxorubicin-induced cardiomyopathy from the cardiotoxic mechanisms to management. Prog Cardiovasc Dis. 2007;49(5):330-352.

6. Swain SM, Whaley FS, Ewer MS. Congestive heart fail- ure in patients treated with doxorubicin: a retrospective analysis of three trials. Cancer. 2003;97(11):2869-2879.

7. Steinherz LJ, Steinherz PG, Tan C. Cardiac failure and dysrhythmias 6-19 years after anthracycline therapy: a series of 15 patients. Med Pediatr Oncol. 1995;24(6):352361.

8. Ganz WI, Sridhar KS, Ganz SS, Gonzalez R, Chakko S, Serafini A. Review of tests for monitoring doxorubicininduced cardiomyopathy. Oncology. 1996;53(6):461470.

9. Ewer MS, Benjamin RS. Complications of cancer and its treatment. In: Holland Jf, Frei E III, Kufe DW, Weichselbaum RR (eds). Cancer Medicine. 4th ed. Williams \& Wilkins, 1997;3204-3215

10. Arico M, Pedroni E, Nespoli L, Vigano M, Porta F, Burgio GR. Long term survival after heart transplantation for doxorubicin induced cardiomyopathy. Arch Dis Child. 1991;66(8):985-986. 